Abstract

\title{
Entropic Transport in Confined Soft-Matter and Biological Systems ${ }^{+}$
}

\section{J. Miguel Rubi}

Department of Condensed Matter Physics, Faculty of Physics, University of Barcelona, Diagonal 647, Barcelona, Spain

† Presented at the Entropy 2021: The Scientific Tool of the 21st Century, 5-7 May 2021; Available online: https://sciforum.net/conference/Entropy2021/.

Published: 5 May 2021

Transport in small-scale biological and soft-matter systems typically occurs under confinement conditions in which particles proceed through obstacles and irregularities of the boundaries that may significantly alter their trajectories. A transport model that assimilates the confinement to the presence of entropic barriers provides an efficient approach to quantify its effect on the particle current and the diffusion coefficient. We review the main peculiarities of entropic transport and treat two cases in which confinement effects play a crucial role, with the appearance of emergent properties. The presence of entropic barriers modifies the mean first-passage time distribution and therefore plays a very important role in ion transport through micro- and nano-channels. The functionality of molecular motors, modeled as Brownian ratchets, is strongly affected when the motor proceeds in a confined medium that may constitute another source of rectification. The interplay between ratchet and entropic rectification gives rise to a wide variety of dynamical behaviors, not observed when the Brownian motor proceeds in an unbounded medium. Entropic transport offers new venues of transport control and particle manipulation and new ways to engineer more efficient devices for transport at the nanoscale.

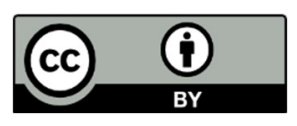

(C) 2021 by the author. Licensee MDPI, Basel, Switzerland. This article is an open access article distributed under the terms and conditions of the Creative Commons Attribution (CC BY) license (http://creativecommons.org/licenses/by/4.0/). 